\title{
GÉNERO, DERECHOS SEXUALES, BIOGRAFÍA Y ESCUELA: ARTICULACIONES Y TENSIONES EN DISCURSOS DE DOCENTES DE ARGENTINA*
}

\author{
ANA LÍA KORNBLIT ${ }^{* *}$ \\ SEBASTIÁN EzEQUiEL SUSTAS ${ }^{* * *}$ \\ PABLO FRANCISCO Di LEO ${ }^{* * * *}$
}

\begin{abstract}
RESUMEN: Presentamos resultados de un proyecto de investigación en el que buscamos analizar los discursos de los docentes en torno a la sexualidad y la recepción de su parte del curso virtual de Educación Sexual Integral, implementado desde el Ministerio de Educación de Argentina, en el que participaron desde 2008 a 2011 más de 3.000 docentes de todo el país. Utilizando técnicas de investigación social cualitativa, analizamos las participaciones de docentes en los foros virtuales de dicho curso durante las ediciones 2009 y 2010. Identificamos las principales categorías emergentes de las reflexiones y relatos biográficos de los docentes alrededor de las dimensiones de género, derechos sexuales, el rol de la escuela en la educación sexual y la visión de sus propias experiencias biográficas.
\end{abstract}

Palabras clave: Género. Sexualidad. Docentes. Capacitación. Educación sexual.

\section{GENDER, SEXUAL RIGHTS, BIOGRAPHY AND SCHOOL: ARTICULATIONS AND TENSIONS IN TEACHERS' DISCOURSE in Argentina}

\begin{abstract}
We present the results of a research project in which we analyze the discourses of schoolteachers around sexuality and their response to the online course Comprehensive Sex Education, implemented by the Argentine Ministry of Education. The course was attended by over 3.000 schoolteachers from all over the country during the period 2008-2011. By using
\end{abstract}

\footnotetext{
* El proyecto de investigación en el que se enmarca este trabajo fue financiado por la Agencia Nacional de Promoción Científica y Tecnológica. En el análisis de los datos contamos con la colaboración de los integrantes del Programa de Educación Sexual Integral del Ministerio Nacional de Educación de Argentina.

** Facultad de Ciencias Sociales de la Universidad de Buenos Aires. Buenos Aires - Argentina.

*** Facultad de Ciencias Sociales de la Universidad de Buenos Aires. Buenos Aires - Argentina.

**** Facultad de Ciencias Sociales de la Universidad de Buenos Aires. Buenos Aires - Argentina.

Contato com os autores: <alkornblit@gmail.com>
} 
qualitative social research techniques, we analyzed the participation of the schoolteachers in the virtual forums of the online course during the 2009 and 2010 editions. We identified the main categories which emerged from the teachers' reflections and biographical stories around the dimensions of gender, sexual rights, the role of schools in sex education, and their view on their own biographical experiences.

Key words: Gender. Sexuality. Schoolteachers. Instruction. Sex education.

GENRE, DROITS SEXUELS, BIOGRAPHIE ET ÉCOLE:

ARTICULATIONS ET TENSIONS DANS LES DISCOURS DE PROFESSEURS en Argentine

RÉSUMÉ: Nous présentons les résultats d'un projet de recherche dans lequel nous analysons les discours des enseignants autour de la sexualité et de la réception de leur part du cours virtuel Éducation Sexuelle Intégrale, mis en œuvre par le Ministère de l'Éducation de l'Argentine, y ont participé entre 2008 et 2011 plus de 3000 enseignants de tout le pays. En utilisant des techniques de recherche sociale qualitative, nous avons analysé les participations des enseignants dans les forums virtuels de ce cours pendant les éditions de 2009 et 2010. Nous avons identifié les principales catégories émergentes des réflexions et histoires biographiques des enseignants autour des dimensions de genre, droits sexuels, le rôle de l'école dans l'éducation sexuelle et la vision de leurs propres expériences biographiques.

Mots-clés: Genre. Sexualité. Enseignants. Formation. Éducation sexuelle.

\section{Introducción}

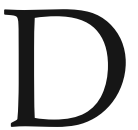

esde la década de 1970 las ciencias sociales han abordado las temáticas de sexualidad con diferentes grados de intensidad. En su mayoría lo han hecho desde los modelos socioconstructivistas, oponiéndose así a los enfoques esencialistas, que rubricaban las explicaciones del sentido común sobre estos temas. El socioconstructivismo se opone a considerar el comportamiento sexual como un fenómeno universal, biológico e individual, enfatizando que se trata de un fenómeno social que se constituye históricamente y por lo tanto que existen múltiples sexualidades. Durante la última década estas concepciones han trasvasado los límites de la academia para difundirse, aunque de modos limitados, en la opinión pública, intentando dar cuenta de los cambios que se han producido paralelamente en las prácticas sexuales, especialmente de los jóvenes. ${ }^{1}$ El Programa de Educación Sexual Integral del Ministerio Nacional de Educación de Argentina es una manifestación de la necesidad de insertar en la escuela una visión de la sexualidad acorde al cambio de paradigma mencionado.

A partir de la aprobación de la Ley n. 26.150, que estableció en Argentina la obligación del Estado en cuanto a garantizar el derecho de niños, niñas y jóvenes a 
recibir Educación Sexual Integral en todos los establecimientos educativos del país, el Consejo Federal de Educación aprobó en mayo de 2008 los lineamientos de Educación Sexual Integral, que definen el piso común de contenidos curriculares válidos para todos los niveles del sistema educativo nacional, en todas sus jurisdicciones. Dicho documento contempla los siguientes puntos centrales:

- La perspectiva de los derechos humanos en relación con la sexualidad.

- La noción de sexualidad comprendiendo aspectos biológicos, psicológicos, sociales, afectivos y éticos y no su asimilación a la genitalidad.

- La superación de los reduccionismos disciplinares, proponiendo por el contrario un abordaje que contemple las mediaciones socio-históricas y culturales, los valores compartidos y las emociones y sentimientos que intervienen en el cuidado y el disfrute de los vínculos con los otros, respetando el propio cuerpo y el cuerpo de los otros.

- La promoción de aprendizajes en los niveles cognitivo, afectivo y ético que posibiliten el trabajo sobre los prejuicios y las creencias que sostienen actitudes discriminatorias.

Como parte de las actividades que el Programa de Educación Sexual Integral del Ministerio comenzó a desarrollar en respuesta a la reglamentación de la Ley n. 26.150, se diseñó un curso virtual que en 2008 estuvo dirigido a docentes del nivel medio y en los años sucesivos a docentes de los diferentes niveles educativos. El curso contempla el desarrollo de cuatro módulos: Introducción a la sexualidad y Educación Sexual Integral; Infancias, adolescencias y derechos; Salud, sexualidad y prevención y Educación Sexual Integral en la escuela. Los cursantes (en número de aproximadamente 1.500 por curso, divididos en 20 aulas virtuales según el nivel educativo en el que se desempeñan) deben participar en los foros virtuales, que son coordinados por un tutor, quien dinamiza el proceso educativo y guía la lectura y discusión del material bibliográfico. Los foros representan un espacio de discusión y encuentro de los cursantes a partir de los temas clave aportados en el aula.

En este trabajo analizamos la producción discursiva de los docentes en los foros, en el supuesto de que ella representa su elaboración del material teórico provisto en el curso, mediada por sus concepciones previas acerca de los temas. Es decir, los comentarios que escriben en los foros no pueden pensarse únicamente como resultado de la incorporación que ellos realizan de los materiales de lectura, sino como el diálogo entre lo nuevo y sus concepciones previas. Es así que nos interesó investigar específicamente en qué medida las concepciones acerca de la sexualidad transmitidas en el curso abonan posturas previas similares por parte de los docentes inscriptos o constituyen insumos claramente diferenciados en relación con los 
bagajes previos con los que ellos cuentan. Y aquí vale una observación importante: probablemente los docentes inscriptos en los cursos tienen en su mayoría posturas en relación con la temática de la sexualidad más afines con los lineamientos del curso que los que pueden tener los no inscriptos. Es decir, su interés en inscribirse en el curso revela ya una preocupación por el tema, fruto probablemente de reflexiones y cuestionamientos previos.

Se trata entonces de identificar los "temas impuestos" por la época (BOURDIEU, 1982) que, en este caso, hacen coincidir las concepciones previas de los cursantes y el material teórico propuesto, y las ausencias de ciertos temas o dificultades con otros, resabios de épocas anteriores, que se "cuelan" en el discurso. Marc Angenot (2010, p. 73) se refiere a esto mismo al expresar:

[...] el observador es sorprendido por el hecho de que lo que para su generación se ha convertido en probable o evidente parece literalmente informulable para los "mejores espíritus" de la generación pasada, que ante ciertos problemas dan prueba de una ceguera colectiva que puede parecer grotesca. El observador queda atrapado aquí en la ilusión de un "progreso ideológico" cuyos obstáculos objetivos son las ideas recibidas del pasado. Estos tabúes universales, por definición no percibidos, han sido distinguidos de las tabúes "oficiales".

Nuestro propósito es poner de manifiesto dichos "tabúes universales" en el corpus constituido por las participaciones de los cursantes en los foros. Con dicho fin, analizamos la participación de los 1.500 docentes que cursaron durante 2009 y 2010 el mencionado curso. Los foros son espacios en los que los cursantes deben expresar sus opiniones con respecto al material de lectura ofrecido en 4 módulos y contestar a las consignas específicas transmitidas para cada módulo.

Para el análisis del corpus seguimos los lineamientos generales de la teoría fundamentada. Aplicando los criterios de parsimonia - maximizar la comprensión de un fenómeno con el mínimo de conceptos posible - y de alcance - ampliar el campo de aplicación del análisis sin desligarse de la base empírica -, en diálogo con el estado del arte y el marco conceptual, fuimos identificando las categorías emergentes de los discursos de los docentes en torno a los lineamientos curriculares mencionados más arriba.

En lo que sigue presentaremos los aspectos más consensuados por los docentes y en ocasiones las voces disidentes, relativos a cada una de las categorías identificadas a través de la aplicación del programa Atlas.ti. El análisis de lo hallado para cada categoría es precedido por algunas referencias teóricas acerca de cada tema, que reflejan el pensamiento actual sobre él. Esto nos permitirá cotejar los dichos de los docentes con el tratamiento que los teóricos de la educación han hecho sobre cada uno de los temas o categorías. 
Organizamos la exposición de las categorías en dos grandes dimensiones identificadas en las intervenciones de los docentes: 1) los temas que se encuentran en la agenda actual, que conforman los contenidos principales del curso, que han sido incorporados por los docentes como el "discurso políticamente correcto"; 2) los aspectos vinculados con sus experiencias, tanto en lo personal como las que surgen de sus trayectorias profesionales e institucionales (ya sea en la formación o en servicio). En las conclusiones reflexionamos en torno a las tensiones entre ambas dimensiones y sus implicancias para la práctica educativa.

\section{Temas vinculados con la agenda pública}

\section{Género}

A partir de nuestro análisis de los discursos docentes identificamos como categorías emergentes algunos de los aspectos que están contenidos en la tradición conceptual de los estudios de género, y que fueron desarrollados en los contenidos del curso: a) el esfuerzo por deconstruir el sexismo, es decir, los estereotipos ligados a la identidad sexual; b) la crítica de la normatividad acerca de la heterosexualidad, acompañada por la aceptación de las sexualidades no hegemónicas. Asimismo, nos parece importante señalar la ausencia en las intervenciones en los foros de un tercer aspecto planteado en los desarrollos conceptuales sobre género: el reconocimiento de los factores de poder que afianzan la supremacía de los hombres por sobre las mujeres.

\section{La deconstrucción del sexismo}

La mayor parte de los docentes tienen clara la existencia de estereotipos en relación con los rasgos genéricos atribuidos a cada uno de los sexos. Esto está particularmente presente entre los docentes de educación inicial, que son quienes se sienten con mayor responsabilidad en cuanto a la posibilidad de "moldear" tales diferencias, en tanto son aspectos que comienzan a instalarse entre los niños que asisten al jardín de infantes. Están, por lo tanto, muy atentos a captar en sí mismos actitudes que refuercen el sexismo, para no incrementar las influencias que los niños reciben de sus familias en este sentido y que traen a la escuela.

Esta postura crítica alcanza también a la caracterización habitual del rol docente en su afinidad con atributos tradicionalmente femeninos: afectividad, irracionalidad, dependencia, continuidad mujer-madre-maestra y los lleva a valorar la elección masculina de la profesión, especialmente en los primeros niveles de la educación. ${ }^{2}$ Como afirman algunos de estos docentes, la vigilancia sobre sus actitudes y conductas en lo relativo a los estereotipos de género requiere que adopten 
sobre sí mismos una mirada de "extranjero", capaz de desnaturalizar las elecciones valorativas y prejuiciosas del sentido común, capacidad ésta que admiten que sólo se adquiere con la práctica y con ejercicios en los que no han sido "entrenados". Por el contrario, como dice una docente, la mirada del sentido común tiene "varias capas geológicas de acumulación"; ejercer su crítica constituye un desafío que muchos de los docentes de nivel inicial son conscientes que quieren asumir.

\section{Crítica de la heteronormatividad}

La crítica a los estereotipos ligados al género lleva a muchos docentes a cuestionarse en qué medida ellos inducen o no la asunción de ciertos atributos tradicionales de los roles femenino o masculino por parte de los niños, condicionados por los "mandatos familiares" ("¿cuánto los estimulamos a variar?"), y cuánto los docentes toleran o no apropiaciones de parte de los alumnos diferentes a las esperables en relación con la heteronormatividad.

Las lecturas y las actividades del curso llevan a un indudable cuestionamiento de parte de los docentes, especialmente, reiteramos, del nivel inicial, en cuanto a qué conductas están dispuestos/as a alentar en relación con las diferencias de género.

Son los docentes de este nivel los que manifiestan también más claramente la preocupación con respecto a las expresiones de los niños contrarias a la heterosexualidad: “¿qué hacer frente al niño con actitudes femeninas y viceversa?”. A pesar de los mayores niveles de aceptación de la homosexualidad que se registran en los foros en relación con relevamientos anteriores realizados por nuestro equipo de trabajo (KORNBLIT; MENDES DIZ, 1994; KORNBLIT et al., 2005; 2006), probablemente a partir de la puesta en agenda de los derechos de las minorías sexuales que se dio a partir de las discusiones y de la aprobación de la ley sobre matrimonio igualitario durante 2010, las dudas expresadas muestran que dicha aceptación es parcial.

La pregunta acerca de si deberían "hacer algo" frente a la manifestación de expresiones contrarias a la heterosexualidad (por lo menos en cuanto a los rasgos tradicionales ligados al género) revela, por un lado, el rechazo a este apartamiento de la normatividad sexual y, por otro, la pretensión de modificarla, acompañada de la idea de poder lograrlo. El plantearse "por lo menos poner al alcance de estos niños la diversidad de elecciones de ambos géneros" sería un modo de acallar un mandato moral que indica que la "desviación" es censurable y que está en sus manos "hacer" algo al respecto.

En este sentido, existen voces que reconocen que las disidencias con la heteronormatividad ponen en crisis el modo de entender el mundo y las relaciones sociales a partir de clasificaciones de personas que las despojan de derechos y las confinan 
a la anormalidad. Estas voces plantean que el axioma de que "el cuerpo es naturaleza" lleva a la afirmación de que existen dos cuerpos a los que les corresponden dos géneros y una sola dirección correcta del deseo: la heterosexual. Lo contrario implica, para algunos participantes, sobre todo respetar las diferencias, apelando a la solidaridad y no a la "lástima" y aceptando que las subjetividades construidas contemplando tales diferencias se han desarrollado a partir de múltiples situaciones.

\section{Derechos sexuales}

La perspectiva de los derechos de los niños y los jóvenes se fue abriendo camino entre los docentes del país a partir de la vuelta a la democracia de 1983. El hecho de que muchos de ellos se formaran como docentes bajo la dictadura y de que en general sus experiencias escolares transcurrieran en climas si no represivos al menos autoritarios, condiciona el que se trate de una perspectiva que debió ser incorporada como algo diferente a lo vivido durante su infancia y juventud. Esto implica que se trata de una incorporación que debió ser hecha en primera instancia desde lo intelectual, para luego vivenciarla en el aula, por lo que cabe, en opinión de muchos docentes, seguir encontrando espacios escolares en los que se vulneran los derechos de los y las alumnos/as.

La dimensión de los derechos sexuales se expresa en los discursos de los docentes a través de dos categorías principales, presentadas como tensiones: a) uniformización versus diferencias; b) sometimiento a la autoridad versus autonomía.

\section{Uniformización versus diferencias}

Muchos docentes perciben que estos dos aspectos se articulan: la fuerte "impronta disciplinadora" de la escuela encuentra su razón de ser en la tendencia "uniformizante y homogeneizadora", que deja poco lugar a la promoción de los derechos, no en sentido declamatorio, sino en lo que cada persona como sujeto de derecho necesita: la igualdad de oportunidades con equidad.

El derecho a la participación y a la expresión de opiniones es reconocido como tal, pero en ocasiones se piensa que no se va más allá de su enunciación, sin que esto implique modificaciones en el modus operandis de las aulas, dado que existen dudas en cuanto a si se generan las condiciones para que todos los alumnos participen efectivamente, "de acuerdo a las posibilidades de cada uno o una". Muchas veces entonces se verifica, en opinión de los cursantes, "una ruptura en el escenario escolar entre el discurso y las prácticas".

El derecho a la información por parte de los alumnos es lo que los docentes reconocen en primer lugar, seguido del derecho a tener experiencias formativas que 
impulsen el respeto por sí mismo y de los otros, en lo cual la educación sexual tiene un rol fundamental. La consideración de los contextos sociales y culturales en los que se desarrolla la vida de los alumnos forma parte de sus derechos, por lo que algunos docentes reconocen que pretender que reproduzcan el perfil de alumnos de sus propias épocas escolares implica una vulneración de sus derechos.

\section{Sometimiento a la autoridad versus autonomía}

Hablar de los derechos de los niños y jóvenes implica fundamentalmente considerar que ellos tienen autonomía para expresar sus necesidades, intereses y deseos, sin que estas instancias puedan ser anticipadas por el supuesto saber del docente. Esto implica dejar de considerar a los alumnos como "los adultos que alguna vez llegarán a ser", para verlos como sujetos que viven un presente, en contextos específicos, con sus propias necesidades, potencialidades, capacidades y expectativas de vida. El proceso de conocimiento de los alumnos como "otros" implica la necesidad de escucharlos, de "habilitarles la palabra", dando lugar al diálogo, y el prestar atención especialmente a los contextos y vínculos particulares que componen su entramado vital.

La formación en cuanto al desarrollo de la conciencia crítica es vista también como una instancia capaz de evaluar la posible presión del grupo de pares en relación con la adopción de conductas que pueden no ser libremente elegidas.

\section{Aspectos vinculados con experiencias personales y profesionales}

\section{Experiencias biográficas}

El análisis de las biografías escolares de los docentes ha sido objeto de consideración en las últimas épocas, a partir del reconocimiento de que las experiencias marcan a las personas y de que la escuela tiene un peso trascendente en la construcción de subjetividades. La no revisión de ciertas prácticas incorporadas desde la propia experiencia escolar puede llevar a que, a partir de su naturalización, se exprese activamente lo vivido pasivamente, en especial en lo que atañe a perpetuar actitudes en las que los derechos de los alumnos no tienen cabida.

La consideración de las biografías escolares de los docentes busca identificar las huellas comunes que dejó en ellos su tránsito por la escuela. Es esta escuela "vivida" lo que es plausible que se reproduzca en la práctica profesional de los docentes, si bien ella es reprocesada en la formación docente. Es importante que los docentes puedan reflexionar sobre ambas experiencias, la de alumnos y la de docentes en formación, induciendo una reflexión sobre el pasado que permita reinterpretar lo 
vivido e identificar las enseñanzas implícitas incorporadas (JACKSON, 1992), que dan cuenta en buena medida de las marcas e improntas que se transforman en "experiencias formativas" (LARROSA, 2003), comprendiendo ambos trayectos: los escolares y los de formación propiamente dicha.

Ahora bien, desde el interés por desentrañar los modos como los docentes pueden acercarse a la introducción de los temas vinculados a la sexualidad en el aula, es importante pensar cuáles serían las condiciones para que "en lugar de reediciones se produzcan resignificaciones de lo vivido y aprendido" (ALLIAUD, 2007, p. 22), y en este lugar es crucial pensar en cómo se da en el aula el encuentro entre las generaciones de docentes y de alumnos.

El estudio de las generaciones ha puesto de relieve que para caracterizarlas es necesario tener en cuenta tanto aspectos de las trayectorias individuales, como los entramados de estas biografías con la cultura de la época y los espacios en consideración. Las "atmósferas culturales" de las que habla Jesús Martín Barbero (1998), para referirse a los particulares climas sociales que caracterizan a una época, adquieren particular significación para entender los desencuentros entre generaciones que se dan muchas veces en el escenario de las escuelas.

Las principales categorías emergentes del análisis de los discursos de los docentes en torno a los aspectos biográficos se refieren a: a) sus experiencias de socialización en la sexualidad; $b$ ) las diferencias generacionales entre dichas experiencias y las de los jóvenes en la actualidad.

\section{La socialización en la sexualidad}

La mayor parte de los docentes relata inicios traumáticos en las dimensiones vinculadas con la sexualidad (menarca, información sobre relaciones sexuales y sobre embarazos y nacimientos, entre otros). En muchos casos, el relato incluye la queja con respecto a la ausencia de la familia como transmisora de información y como contenedora frente a los cambios corporales asociados a la pubertad, lo que deriva en una concepción de la sexualidad ligada a miedos y a opresión. El pudor y la vergüenza resultantes de tales experiencias tienen que ver seguramente con el hecho de que en los foros los docentes no hablen, por ejemplo, de dificultades posibles en el inicio de sus relaciones sexuales ni de prácticas sexuales en general. El énfasis está puesto en la dificultad experimentada en su niñez y adolescencia en la vida familiar y en la escuela para "hablar" de los temas sexuales. En algunos casos relatan que esto se suplía con la observación en la vida rural de los ciclos sexuales de los animales, lo que rubrica la tendencia a enfatizar los aspectos biológicos de la sexualidad ("se nos quedaban afuera la afectividad, los derechos, el compartir unos con otros la vida"). 
Las décadas de autoritarismo político en las que transcurrieron para la mayor parte de los cursantes sus etapas de escolarización dejaron obviamente su impronta en relación con el ejercicio de su rol. Sólo tardíamente, para muchos de ellos, surgió el cambio de paradigma por el que fue posible el debate. Uno de los docentes lo expresa de un modo impactante: "[...] y hasta comenzó a haber algunos docentes que se interesaban por nosotros". Como es lógico, en tal panorama, la educación sexual formaba parte de lo que otro docente, apelando a una categoría de las Ciencias de la Educación, denomina "currículo nulo", ${ }^{3}$ sólo contrarrestado en parte por algunos profesores de Biología que se "animaban a tratar el tema" desde sus raíces en la asignatura que dictaban. Para muchos, el peso de las décadas de oscurantismo tiene que ver con los vestigios de esas posturas en temas como la sexualidad.

La posibilidad de encarar los temas vinculados con la sexualidad en la escuela debe realizarse, pues, sin haber tenido la experiencia de haber atravesado por su aprendizaje en el ámbito escolar, y la mayor parte de las veces sin haberlo hecho tampoco en el ámbito familiar. Los pares en una primera aproximación y figuras adultas de "pensamiento abierto", a las que se agradece, o búsquedas de información a través de lecturas son las fuentes de aprendizaje mencionadas por una gran mayoría, de lo que queda la sensación de haber tenido que recorrer un camino "en soledad".

También surgen en relación con este tema críticas en relación con el mundo de sus adultos en cuanto a muchas explicaciones tradicionales sobre la gestación y el nacimiento de los niños que "[...] han sumergido a la infancia en la incomprensión y han expuesto a niñas y niños a modos de configuración de su sexualidad bastante terroríficos". El hecho de la mayor porosidad de las fronteras entre la familia y la escuela, por un lado, y por otro los diferentes estímulos que les llegan a los niños y jóvenes desde los medios de comunicación y otras fuentes de información hacen que en este sentido ellos sean menos dependientes de lo que eran sus docentes con respecto a las mismas instituciones, cuando tenían la edad de sus alumnos. Esto también ha obligado a un reacomodamiento de parte de los docentes, quienes deben aceptar que comparten mucho más que antes con otras instancias las fuentes de las que los alumnos aprenden.

\section{Diferencias generacionales}

La escisión entre reproducción y sexualidad, los cambios en lo que respecta al matrimonio, la mayor aceptación de familias no tradicionales, el mayor reconocimiento de las diversidades sexuales, el adelantamiento del inicio de las relaciones sexuales son los "cambios epocales" más importantes en lo que respecta a la esfera sexual. Estos cambios son registrados y aceptados o no por los docentes, pero en todos los casos son 
mencionados como instituyendo una distancia entre las experiencias vividas por ellos en relación con la esfera sexual y las vividas por sus alumnos. Aun cuando en algunos casos afirman que tienen una visión de la sexualidad en toda su complejidad, lo que aparece es una suerte de "quiebre" entre cómo la viven los jóvenes y los adultos. Especialmente para los jóvenes de los sectores sociales más vulnerables, los docentes perciben que ella está teñida por situaciones que connotan dificultades y riesgos: embarazos, problemas de salud, entre otros, situaciones teñidas de aspectos negativos que contribuyen o se originan en la desigualdad social, lo que muchas veces quita espacio a otras muchas visiones posibles del tema.

Quizás la diferencia más importante que registran los docentes entre sus propias experiencias como alumnos y las vividas por quienes ahora son escolares es el que se haya instalado, por lo menos teóricamente, el derecho de los niños y jóvenes a plantear sus necesidades y deseos, mientras que, tal como lo expresa uno de los cursantes, "jamás en mi infancia pude expresarme de esa manera, sólo el adulto tenía derecho a la palabra, y esa palabra era ley". Desandar lo vivido, entonces, lo que implica a la vez abrir espacios de diálogo con los alumnos y enfocar el tema sin prejuicios y estereotipos incorporados, conlleva un desafío reconocido por muchos de los cursantes.

\section{Trayectorias profesionales e institucionales en torno a la educación sexual}

En relación a las experiencias profesionales e institucionales de los docentes en torno a la educación sexual, identificamos las siguientes categorías emergentes: a) la hegemonía de una concepción biologista en torno a la sexualidad; b) el temor al desborde en el abordaje de estas temáticas en la escuela; c) obstáculos y facilitadores para el desarrollo de estrategias de educación sexual integral.

\section{Hegemonía de la concepción biologista}

Muchos programas de educación sexual tienen como eje el modelo biologista, que se centra en la transmisión de información sobre anatomía y fisiología del aparato genital, por lo tanto, el tratamiento del cuerpo se reduce a la reproducción y a las relaciones sexuales genitales, dejando de lado todo lo vinculado con la afectividad y con las relaciones humanas, por un lado, y con lo histórico-cultural por otro. Se abre el camino así a la perspectiva médica y a la validación del papel de los especialistas como recurso escolar para el tratamiento de los temas vinculados con la sexualidad. Tal perspectiva amplió su injerencia en la escuela a partir de la preocupación por el embarazo adolescente y por el VIH/sida, que robustecieron la idea de la necesidad de llevar a la escuela el enfoque preventivista. Este enfoque gira en torno a las amenazas de las enfermedades y de los efectos no deseados de la sexualidad, dejando 
de lado no sólo las relaciones humanas sino además todo lo atinente al deseo como parte de la sexualidad y a su importancia en términos del proceso de subjetivación humana. La no consideración del deseo en el abordaje de la sexualidad lleva a plantear la experiencia sexual humana de un modo empobrecido, presentándola desde afuera de la experiencia juvenil y docente (MORGADE, 2011).

Las encuestas realizadas por los cursantes a otros docentes acerca de qué es la sexualidad para ellos (tarea encomendada en el curso) revelan una posible distancia entre los que se anotaron para seguir el curso de ESI y los que no, dado que en todos los casos las respuestas recogidas hablan del predominio de la concepción biologista, que considera a la sexualidad como un proceso anátomo-fisiológico en lo que atañe a la genitalidad y a la reproducción, en algunos casos acompañado por una visión médico-sanitarista, que vincula la sexualidad con la prevención de enfermedades, o por una visión psicologicista. El gran ausente según sus reflexiones es, pues, el contexto social.

Siguiendo a Morgade (op. cit.), la prevención a la que se apunta desde el enfoque médico-biológico no es subjetivante, es decir, no alienta la incorporación de un saber que incide en el "ser" de los destinatarios, sino que permanece como una información en el plano de lo cognitivo, sin vincularse con la experiencia y la afectividad. Como dice la autora citada, el recurso de parte de los docentes a la "expertez" de los profesionales del área de salud, legitimados en su saber por sus conocimientos y por su rol, implica el reconocimiento de una carencia: la de la posibilidad de contar con la propia experiencia y el propio recorrido desde la afectividad.

Las dimensiones emocional y social como constitutivas de la sexualidad están presentes, por lo menos teóricamente, en la concepción de los cursantes sobre el tema, muchos de los cuales plantean que la educación sexual está en relación con los derechos de los/las niños/as y adolescentes, con la equidad de género, con la aceptación de la diversidad, con la autoestima, con "el conocimiento y la apropiación de pautas que generen autocuidado para prevenir posibles abusos", con "el entramado de relaciones sociales a lo largo de la vida".

A pesar de ello, la visión biológica, junto con la expectativa de poder delegar el tratamiento de los temas vinculados con la sexualidad han hecho que se valorara la "expertez" del personal de salud para encararlos en la escuela. El tema "se tercerizó" con los profesionales de la salud con la pretensión de darle así un carácter "más objetivo" y un propósito "preventivo/correctivo". Sin embargo, la ampliación del concepto de salud como "nudo conceptual abordable desde muchos campos disciplinares y desde muchas dimensiones de la vida" hace que, para muchos cursantes, las cuestiones vinculadas con la sexualidad deban pensarse desde el ámbito de la educación y no desde el médico. 


\section{El temor al desborde}

¿Qué es el desborde para los docentes? El recorrido por sus expresiones en los foros analizados muestra que sus temores respecto a dar respuestas inadecuadas o a cuestionarse a sí mismos como "no estando a la altura de las circunstancias" tienen que ver con recibir preguntas o inquietudes de los alumnos que no se han planteado para sí mismos o para las cuales no tienen respuestas, es decir, evidenciar que no se cuenta con el supuesto saber que ellos mismos descuentan que deberían tener, sin poder o querer blanquear el desconocimiento o la duda.

Las dudas pasan también por el cuestionamiento acerca de cuál es el grado de "confianza" entre alumnos y docentes que permita, a la vez que integrar la afectividad, no ser invadido por sus diferentes manifestaciones al punto de que ellas entorpezcan el proceso de enseñanza-aprendizaje. La doble acepción del término en cuanto a creer en el otro sin presumir de su parte posibles malos tratos y el sentimiento de acortamiento de distancias interpersonales (la "familiaridad"), que implica el "entrar en confianza", confluyen en la "garantía de intimidad que implica como mínimo, respeto por; en lo posible, espacio para" (DÍAZ VILLA, 2011, p. 90). El temor a no darse cuenta de cuándo hay que establecer límites que no es aconsejable traspasar, pero a la vez abrir espacios que permitan plantear preocupaciones, es frecuente entre los docentes.

Otra zona gris a la que temen los docentes, especialmente los de nivel inicial, es la que tiene que ver con no poder precisar cuál es el grado de información adecuada según la edad de los niños y sus desarrollos socio-afectivo y cognitivo, dado que el "exceso de información" puede ser tan "perjudicial" como la falta de ella. A esto se agregan, particularmente también en los docentes de este nivel, las tensiones en relación con qué es lo que la familia acuerda que se trate en el aula.

La falta de un saber consensuado con otros docentes, surgido de espacios de reflexión comunes, hace también que muchos sientan que se mueven en soledad, por añadidura, "sin un marco teórico que sustente la práctica en relación con estas cuestiones".

A esto se agrega la percepción de no haber desarrollado, ni en su escolaridad ni en su formación, habilidades para la verbalización de sentimientos, necesidades y problemas.

\section{Obstáculos y facilitadores para el desarrollo de estrategias de educación sexual integral}

Las menciones de los cursantes con respecto a los factores que pueden influir en el desarrollo de su parte de estrategias de aplicación de la educación sexual integral en el aula pueden clasificarse en obstáculos y facilitadores de las mismas. Los 
obstáculos que mencionan son en primer lugar la falta de acompañamiento de las autoridades de la escuela o incluso su oposición y en relación con esto, el que la ESI no tiene en general en las escuelas la jerarquía de proyecto institucional, lo que haría que formara parte de los lineamientos curriculares sistemáticos.

Por otra parte, el hecho de que sea aún un tema sobre el que no existe consenso entre los adultos, especialmente otros docentes y, en algunos casos, familiares, conspira también contra su aplicación de forma sistemática. Asimismo, también es evaluado como un obstáculo el sistema educativo, que exige calificar numéricamente saberes que son trabajados holísticamente, que tienen que ver con la formación de las personas.

Otro de los obstáculos identificados es la dificultad de la institución educativa para incorporar una concepción ampliada de la salud como dimensión que incluye el desarrollo potencial de las capacidades de un sujeto en un medio social que las habilite y no solamente lo concerniente a la ausencia de enfermedades.

El paradigma de la salud en sentido amplio requiere, según algunos docentes, que se trabaje no en función de la transmisión de información, del docente al alumno, sino como una construcción que el sujeto alumno hace de su realidad vivida, en un proceso de interacción con el sujeto docente.

La dificultad de la escuela para abrirse a modelos alternativos a los hegemónicos en relación con la salud, que han tenido una fuerte inserción social, es señalada por un docente que se interroga por qué no se ha incorporado en el programa escolar disciplinas como el yoga o el tai-chi, por qué no hay talleres de cocina natural, por qué no se modifica la oferta de los kioscos de las escuelas, introduciendo golosinas no tradicionales, etc.

En relación con el marco general que facilitaría la aplicación de la ESI, se mencionan los siguientes aspectos:

- La creación de espacios de reflexión para docentes, que posibiliten el trabajo sobre los propios estereotipos y permitan visibilizar lo que les pasa como adultos en relación con el tratamiento de la temática en el aula.

- La modalidad participativa de enseñanza, con propuestas que consideren a los alumnos como sujetos de derechos y docentes abiertos a la escucha, lo que posibilita rescatar las ideas previas que poseen los alumnos y las asociaciones que realizan con los nuevos contenidos que están asimilando.

- El logro de un clima de respeto mutuo en el aula, que permita entablar lazos de confianza entre alumnos y docentes. 
- El trabajar teniendo en cuenta la afectividad, poniendo en la mira el reforzamiento de la autoestima, la autoreflexión y el proyecto de vida.

- La posibilidad de revisar las prácticas cotidianas entre colegas docentes, de modo de favorecer la reflexión compartida y el autoanálisis como único medio para desnaturalizar prácticas incorporadas acríticamente, fuertemente arraigadas, y que se piensan con las "mejores intenciones", "por el bien del otro".

- La lectura y análisis de marcos teóricos que permitan la búsqueda de estrategias didácticas pedagógicas adecuadas a estos temas, para cada grupo en particular.

- El convencimiento de la necesidad de transmitir herramientas más que de modelar comportamientos, lo que permitirá vivir la sexualidad como algo en construcción a lo largo de la vida en lugar de prescribir, tratando de lograr un efecto deseable o esperable a través de suministrar la "información correcta".

- En relación con la promoción de la salud, tener en cuenta de modo especial la creación de proyectos que apunten a que los mismos niños/as y adolescentes se responsabilicen, a través de llevar adelante campañas diversas en el ámbito del aula, de la escuela y de la comunidad.

\section{Consideraciones finales}

El análisis de la participación de los docentes en los foros virtuales pone de manifiesto una profunda escisión entre dos grandes dimensiones. Por un lado, las propuestas teóricas transmitidas en el curso retoman en muchos casos concepciones con las que los docentes ya cuentan, a partir de la asimilación que han realizado de lo que denominamos "cambios epocales" en relación con el género y la sexualidad. Sin embargo, por otro lado, en sus reflexiones en torno a experiencias biográficas personales, profesionales e institucionales develan la persistencia de lo que, siguiendo a Angenot (2010), hemos denominado "tabúes universales", expresados principalmente en la hegemonía de una concepción biologista de la sexualidad, en los temores para su abordaje en el contexto escolar y en la ausencia de las temáticas del goce y el placer sexual.

En relación con la temática de género persiste el escotoma acerca de las desigualdades que obstaculizan el advenimiento de las mujeres a la plena ciudadanía, derivado de una distribución asimétrica del poder. La aceptación de las sexualidades no hegemónicas es parcial, dado que algunos docentes cuestionan la posibilidad 
de los alumnos de alejarse de la heteronormatividad en las manifestaciones tempranas de sus elecciones sexuales. Aspectos relativos al carácter relacional y la dimensión de poder del género, en la medida en que es un elemento constitutivo de las relaciones sociales basadas en las diferencias que distinguen a los sexos y una forma primaria de relaciones significantes de poder (SCOTT, 1996), se encuentran prácticamente ausentes de las reflexiones de los docentes en los foros, lo que muestra que no existe una visión de la importancia de la articulación de los factores sociales y políticos con los diferentes significados de la sexualidad. Sólo en contadas ocasiones se hace referencia al "machismo" y a cómo las actitudes englobadas en él conspiran contra la posibilidad del desarrollo de las mujeres como ciudadanas plenas.

En cuanto a los derechos sexuales, el énfasis está puesto en la posibilidad de que los alumnos tengan derecho a la información en materia de sexualidad, para lo que se parte de la necesidad de "escuchar al otro", de "darle la palabra", en un giro desde las relaciones de autoridad y jerárquicas vividas por los docentes durante su propia escolaridad y lo que afirman que debería ser el clima en las aulas en la actualidad, no siempre vigente.

El "tabú oficial" respecto a hablar de la sexualidad, vivido por los docentes en sus etapas formativas, tanto en la familia como en la escuela, oculta el "tabú universal" acerca de las prácticas sexuales como posibilitadoras del goce. A pesar de todas las consideraciones que revelan que buena parte de los cursantes tienen visiones críticas y complejas con respecto a la sexualidad, hay una dimensión que está prácticamente ausente de su discurso y es la que se vincula con el goce y el placer asociado a la posibilidad del disfrute del cuerpo. De tal forma, el placer sexual es el gran ausente, aún en la escuela actual y esto deriva, por un lado, de la prescindencia de los cuerpos y, por otro, de la afectividad, escindida del mundo racional que se supone es el leit motiv de la escuela.

De todos modos hay que rescatar que muchos docentes han adoptado el paradigma de la salud en sentido amplio, condición para un tratamiento de la sexualidad que vaya más allá de sus condiciones biológicas y tome en cuenta los factores históricos y culturales que la enmarcan. Los inscriptos en el curso, al menos, critican la delegación del tema en profesionales de la salud, quienes refuerzan el sentido limitado de la sexualidad como ligada a la genitalidad y a la reproducción. A pesar entonces de que resta incorporar dimensiones importantes en el paradigma de la sexualidad que manejan los docentes, creemos que puede decirse que ha habido un avance.

\section{Notas}

1. Teniendo conciencia de la orientación androcéntrica del español, utilizamos el género masculino en los plurales sólo para facilitar la lectura. 
2. En este sentido, una anécdota curiosa comentada por una participante es que en algunas provincias rige una ordenanza por la que se impide ejercer la docencia a los varones en el nivel inicial.

3. El curriculum nulo, excluido o ausente, según Eisner (1979), consiste en el conjunto de contenidos que no están presentes o lo están de modo insuficiente, en los curricula oficiales, pero cuya inclusión en el proceso de enseñanza-aprendizaje constituye una demanda de los alumnos o de la sociedad.

\section{Referencias}

ALLIAUD, A. La biografía escolar en el desempeño de los docentes. Victoria: Universidad de San Andrés, 2007. (Documentos de trabajo n. 22).

ANGENOT, M. El discurso social. Buenos Aires: Siglo XXI, 2010.

ARRIETA DE MEZA, B.; MEZA CEPEDA, R. El curriculum nulo y sus diferentes modalidades. Disponível em: <http://www.rieoei.org/deloslectores/220Meza.PDF>. Acesso em: 20 jun. 2010.

BLANCO, R.; PIERELLA, M.P. Experiencias estudiantiles en la universidad contemporánea: notas acerca de modos de abordaje de los discursos sobre autoridad, sexualidad y afectividad. Educación, Lenguaje y Sociedad, Santa Rosa, v. 6, n. 6, p. 69-84, 2009.

BOURDIEU, P. ¿Qué significa hablar?: la economía de los intercambios lingüísticos. Madrid: Akal, 1982.

DELORENZI, O. Biografía escolar: ¿determinante de las prácticas docentes o punto de partida para su construcción? Voces de la Educación Superior, Publicación Digital n. 2, DGCyE. Disponível em: <http://abc.gov.ar/lainstitucion/revistacomponents/revista/ archivos/voces/numero01/ArchivosParaImprimir/1_.pdf>. Acesso em: 20 jun. 2010.

DÍAZ VILLA, G. En la escuela no tenemos la confianza. In: MORGADE, G. (Coord.). Toda educación es sexual. Buenos Aires: La Crujía, 2011. p. 81-102.

EISNER, E.W. The educational imagination. New York: MacMillan, 1979.

JACKSON, P.W. La vida en las aulas. Madrid: Morata, 1992.

KORNBLIT, A.L.; MENDES DIZ, A.M. Modelos sexuales en jóvenes y adultos. Buenos Aires: Centro Editor de América Latina, 1994.

KORNBLIT, A.L. et al. Salud y enfermedad desde la perspectiva de los jóvenes. Un estudio en jóvenes escolarizados de la Ciudad de Buenos Aires. Buenos Aires: Instituto de Investigaciones Gino Germani, 2005. (Documento de trabajo n. 45).

KORNBLIT, A.L. et al. Salud y enfermedad desde la perspectiva de los jóvenes. Un estudio en jóvenes escolarizados de todo el país. Buenos Aires: Instituto de Investigaciones Gino Germani, 2006. (Documento de trabajo n. 46). 
LAMAS, M. (Comp.). El género: la construcción cultural de la diferencia sexual. México, DF: Pueg/Unam; M. Á. Porrúa, 1996.

LARROSA, J. Entre las lenguas: lenguaje y educación después de Babel. Barcelona: Laertes, 2003.

MARTÍN BARBERO, J. Experiencia audiovisual y desorden cultural. In: MARTÍN BARBERO, J.; DE LA ROCHE, F.L. (Ed.). Cultura, medios y sociedad. Bogotá: CES/ Universidad Nacional de Colombia, 1998.

MORGADE, G. (Coord.). Toda educación es sexual. Buenos Aires: La Crujía, 2011.

SCOTT, J. El género: una categoría útil para el análisis histórico. In: LAMAS, M. (Comp.). El género: la construcción cultural de la diferencia sexual. México DF: Pueg/ Unam; M.Á. Porrúa, 1996.

SEPÚLVEDA, M.P.; RIVAS, J.I. Voces para el cambio: las biografías como estrategias de desarrollo profesional. In: SANTOS GUERRA, M.A.; BELTRÁ, R. (Ed.). Conocimiento, ética y esperanza. Málaga: Universidad de Málaga, 2003. p. 367-381.

SIMON, W.; GAGNON, J.H. Sexual scripts: permanence and change. Archives of Sexual Behaviour, v. 5, n. 2, p. 97-120, 1987.

VILLA, A. Cuerpo, sexualidad y socialización. Buenos Aires: Noveduc, 2007.

VILLA, A. (Comp.). Sexualidad, relaciones de género y de generación. Buenos Aires: Noveduc, 2009.

WAINERMAN, C.; DI VIRGILIO, M.M.; CHAMI, N. La escuela y la educación sexual. Buenos Aires: Manantial; Universidad de San Andrés; Unfpa, 2008.

Recebido em 22 de junho de 2012.

Aprovado em 13 de setembro de 2013. 